\title{
(6) OPEN ACCESS \\ European consensus conference on faecal microbiota transplantation in clinical practice
}

\author{
Giovanni Cammarota, ${ }_{1}^{1}$ Gianluca laniro, ${ }^{1}$ Herbert Tilg, ${ }^{2}$ Mirjana Rajilić-Stojanović, ${ }^{3}$ \\ Patrizia Kump, ${ }^{4}$ Reetta Satokari, ${ }^{5}$ Harry Sokol, ${ }^{6}$ Perttu Arkkila, ${ }^{7}$ Cristina Pintus, ${ }^{8}$ \\ Ailsa Hart, ${ }^{9}$ Jonathan Segal, ${ }^{9}$ Marina Aloi, ${ }^{10}$ Luca Masucci, ${ }^{11}$ Antonio Molinaro, ${ }^{12}$ \\ Franco Scaldaferri, ${ }^{1}$ Giovanni Gasbarrini, ${ }^{1}$ Antonio Lopez-Sanroman, ${ }^{13}$ \\ Alexander Link, ${ }_{1}^{14}$ Pieter de Groot, ${ }^{15}$ Willem M de Vos, ${ }_{1}^{5,16}$ Christoph Högenauer ${ }^{4}$ \\ Peter Malfertheiner, ${ }^{14}$ Eero Mattila, ${ }^{17}$ Tomica Milosavljević, ${ }^{18}$ Max Nieuwdorp, ${ }^{12,15,19}$ \\ Maurizio Sanguinetti, ${ }^{11}$ Magnus Simren, ${ }^{20}$ Antonio Gasbarrini, ${ }^{1}$ The European FMT \\ Working Group
}

\begin{abstract}
- Additional material is published online only. To view please visit the journal online (http://dx.doi.org/10.1136/ gutjnl-2016-313017)

For numbered affiliations see end of article.
\end{abstract}

Correspondence to

Professor G Cammarota, Gastroenterological Area, Fondazione Policlinico Universitario Gemelli, Università Cattolica del Sacro Cuore, Largo A. Gemelli 8, Rome 00168, Italy; giovanni.cammarota@unicatt.it

Received 7 September 2016 Revised 1 December 2016 Accepted 4 December 2016 Published Online First 13 January 2017
CrossMark

To cite: Cammarota $\mathrm{G}$ laniro G, Tilg H, et al. Gut 2017:66:569-580.

\section{ABSTRACT}

Faecal microbiota transplantation (FMT) is an important therapeutic option for Clostridium difficile infection. Promising findings suggest that FMT may play a role also in the management of other disorders associated with the alteration of gut microbiota. Although the health community is assessing FMT with renewed interest and patients are becoming more aware, there are technical and logistical issues in establishing such a non-standardised treatment into the clinical practice with safety and proper governance. In view of this, an evidence-based recommendation is needed to drive the practical implementation of FMT. In this European Consensus Conference, 28 experts from 10 countries collaborated, in separate working groups and through an evidence-based process, to provide statements on the following key issues: FMT indications; donor selection; preparation of faecal material; clinical management and faecal delivery and basic requirements for implementing an FMT centre. Statements developed by each working group were evaluated and voted by all members, first through an electronic Delphi process, and then in a plenary consensus conference. The recommendations were released according to best available evidence, in order to act as guidance for physicians who plan to implement FMT, aiming at supporting the broad availability of the procedure, discussing other issues relevant to FMT and promoting future clinical research in the area of gut microbiota manipulation. This consensus report strongly recommends the implementation of FMT centres for the treatment of $C$. difficile infection as well as traces the guidelines of technicality, regulatory, administrative and laboratory requirements.

\section{INTRODUCTION}

Faecal microbiota transplantation (FMT) consists of the infusion of faeces from a healthy donor to the GI tract of a recipient patient, in order to treat a specific disease associated with alteration of gut microbiota. A large body of evidence, including randomised controlled trials (RCTs), systematic reviews and meta-analyses, proved clear evidence that FMT is a highly effective treatment against recurrent Clostridium difficile infection (rCDI). ${ }^{1-7}$ Due to the rising prevalence, severity and mortality of this infection, the therapeutic role played by FMT is therefore important to save human lives and to decrease the economic burden on healthcare systems. ${ }^{8-11}$ Based on these data, both the European Society for Microbiology and Infectious Disease and the American College of Gastroenterology recommend FMT as a treatment for rCDI. ${ }^{12} 13$

Beyond the treatment of CDI, FMT has also been investigated in other disorders associated with the alteration of gut microbiota. In particular, studies in humans include RCTs in patients with $\mathrm{UC}$ and metabolic syndrome (MS). ${ }^{14-16}$

The global interest in FMT is increasing, and both doctors and patients are increasingly aware and informed. Although the dissemination of FMT in the clinical practice is restricted by regulatory and bureaucratic issues (principally related to costs, donor programme, safety control), ${ }^{17-19}$ the FMT practice is booming, ranging from highly organised stool banking programmes to individual treatments with patient-identified directed donors, and even to individual and harmful do-it-yourself practices. Working groups (WGs) from the USA, Austria and France released recommendations on indications and methods of FMT. ${ }^{20-22}$ Authoritative published guidelines and recommendations have been released as expert opinions rather than evidence-based consensus reports. A rigorous and formal evidencebased process to drive the wide range of FMT practice has not been performed yet.

The aim of this evidence-based consensus report is to define indications and methodology for the use of FMT in the treatment of CDI, to discuss the suitability of FMT for indications other than CDI and to address the minimum requirements needed to implement a FMT centre. The final aim is to encourage and drive the dissemination of the procedure and to promote further clinical research in the area.

\section{METHODS}

\section{Consensus development process}

The process of development of the consensus conference, aimed at drawing up evidence-based recommendations for the use of FMT in clinical practice, included the following steps: selection of 
the expert panel members; identification of main topics and generation of WGs; development of statements according to the best available evidence; development of consensus through the electronic Delphi process and face-to-face meeting to release the final version of statements.

Consensus presidents (GC, AG) and consensus secretary (GI) chose the consensus members based on their expertise in the field of FMT and/or gut microbiota. A total number of 28 experts from 10 European countries constituted the experts panel and had an active role in the development of consensus process. Each member was assigned, according to her/his expertise, to one of the following five WGs: WG-1, indications; WG-2, donor selection; WG-3, preparation of faecal material; WG-4, clinical management and faecal delivery; WG-5, basic requirements for implementing an FMT centre.

Members of each WG elected a chair to coordinate work and to liaise with the consensus presidents and secretary. For each main topic, the consensus presidents and secretary drew up three to six key issues for which members of the corresponding WG were requested to formulate statements after a systematic review of the literature. The quality of evidence and strength of recommendation for each statement were determined according to the Grading of Recommendations Assessment, Development and Evaluation (GRADE) system. ${ }^{23}{ }^{24}$ Definition of the strength of recommendation is given in table 1 and the quality of the published evidence is defined in table 2 .

Statements from each WG were edited by the respective chair and then uploaded to an online electronic voting system (http:// armstrong.wharton.upenn.edu/delphi2/) by the consensus secretary. The Delphi method was used to achieve a consensus. ${ }^{25}$ Several rounds of statements were uploaded and sent out, and the experts' anonymous responses were collected and shared with the group after each round. The experts were allowed to adjust their answers in subsequent rounds. After multiple rounds, the Delphi method enabled achievement of the 'correct' response through consensus.

For each statement, consensus members were requested to rate their level of agreement, among the following: (1) agree strongly; (2) agree with reservation; (3) undecided; (4) disagree; (5) disagree strongly. If the rating was other than 'agree strongly', respondents were requested to add some comments to explain their reservation/disagreement, and how to improve the statement. For each statement, the pre-established threshold was reached when the overall result was $\geq 80 \%$, with the experts agreeing either strongly or with reservation. All statements not reaching $80 \%$ of agreement were revised and rated again in further round of voting. To reach consensus, two rounds of electronic votings were run overall.

Afterwards, 22 of 28 (79\%) consensus members gathered on 9 July 2016 in Rome for a face-to-face meeting. Before the final conference of the expert panel, each WG had an internal meeting to reach a WG agreement on statements that had not reached consensus at the electronic voting. At the final conference, the chairs presented the respective WG statements to all

Table 1 Definition of the strength of recommendation and statement

\begin{tabular}{ll}
\hline Strength & Definition \\
\hline Strong & Strongly supports a recommendation for use and/or a statement \\
Weak & Marginally supports a recommendation for use and/or a statement \\
\hline
\end{tabular}

Table 2 Definition of the quality of evidence (QoE)

\begin{tabular}{ll}
\hline QoE & Definition \\
\hline $\begin{array}{l}\text { Strong } \\
\text { Moderate }\end{array}$ & $\begin{array}{l}\text { Evidence from at least one properly designed RCT } \\
\text { Evidence from at least one well-designed clinical trial, without } \\
\text { randomisation; from cohort or case-control analytic studies } \\
\text { (preferably from more than one centre); from multiple time series }\end{array}$ \\
Low & $\begin{array}{l}\text { Evidence from opinions of respected authorities, based on clinical } \\
\text { experience, descriptive case studies or reports of expert committees }\end{array}$ \\
\hline RCT, randomised controlled trial.
\end{tabular}

members in the plenary session and the expert panel voted on each statement by a show of hands. Statements not reaching consensus $(<80 \%)$ were discussed and either removed or modified and voted again. Finally, the consensus secretary provided a summary of the finalised statements.

Chairs and all WG members provided commentaries and supporting literature for each statement. Finally, all 28 members of the consensus approved the final version of released statements and commentaries.

\section{RESULTS}

\section{Indications}

Key issue: CDI (clinical pictures of CDI are described in table 3).

\section{FMT for rCDI}

Statement: FMT is recommended as treatment option for both mild and severe rCDI. Its implementation in clinical practice is recommended.

Quality of evidence: high.

Strength of recommendation: strong.

Comment: Infusion of faecal microbiota from a healthy donor into a recipient individual with CDI can restore the healthy microbial flora in the diseased colon, leading to the resolution of symptoms. In two open-label RCTs including

Table 3 Clinical pictures compatible with CDI according to the ESCMID guidelines (modified from Debast et al). ${ }^{13}$

\begin{tabular}{|c|c|}
\hline $\begin{array}{l}\text { Clinical } \\
\text { picture }\end{array}$ & Definition \\
\hline Severe & $\begin{array}{l}\text { Episode of CDI with one or more specific clinical (fever, } \\
\text { haemodynamic instability, respiratory failure which needs } \\
\text { mechanical ventilation, signs and symptoms of peritonitis, signs } \\
\text { and symptoms of colonic ileus), laboratory (marked leucocytosis, } \\
\text { rise in serum creatinine and lactate, marked decrease of serum } \\
\text { albumin), radiological (colon distension, colonic wall thickening) } \\
\text { or endoscopic (pseudomembranous colitis), symptoms and signs } \\
\text { of severe colitis or complicated course of disease }\end{array}$ \\
\hline Recurrent & $\begin{array}{l}\text { When CDI recurs within } 8 \text { weeks after the onset of a previous } \\
\text { episode, provided the symptoms from the previous episode } \\
\text { resolved after completion of initial treatment. It is not feasible } \\
\text { to distinguish recurrence due to relapse (renewed symptoms } \\
\text { from already present CDI) from recurrence due to reinfection in } \\
\text { daily practice }\end{array}$ \\
\hline Refractory & $\begin{array}{l}\text { CDI that is unresponsive to the antimicrobial treatment, namely } \\
\text { persistence of diarrhoea with } C D \text { toxin positive or persistent } \\
\text { diarrhoea with toxin negative in the absence of other possible } \\
\text { causes of diarrhoea (eg, IBS, IBD, non-CDI antibiotic-associated } \\
\text { diarrhoea) }\end{array}$ \\
\hline
\end{tabular}


patients with rCDI, FMT showed significantly higher resolution rates than vancomycin (94\% and $90 \%$ vs $31 \%$ and $26 \%$, respectively). ${ }^{1}{ }^{2}$ Both studies have a small sample of enrolled subjects, as they were stopped early for futility. The excellent efficacy rates (above 80\%) of FMT for the treatment of rCDI confirmed afterwards with a larger double-blind RCT, aimed at comparing frozen FMT with fresh FMT $(83.5 \%$ and $85.1 \%$, respectively at per-protocol analysis). ${ }^{3}$ A further double-blind RCT in which FMT using donor stool (heterologous) was compared with FMT using patient's own (autologous) stool (this latter study was published after the conclusion of this consensus conference). ${ }^{26}$

Moreover, in several systematic reviews and meta-analyses, including both RCTs and non-controlled studies, rCDI resolution rates achieved by FMT ranged between $85 \%$ and $89.7 \% .^{4-6}$

In all these studies, FMT also showed an excellent safety profile, at least in the short-term follow-up, as only few and almost mild adverse events were reported. Currently, long-term safety data are lacking. In theory, it may be possible to transmit potentially harmful microbiota traits, which may be not apparent for decades. This possibility should however be envisaged in the context of a favourable risk-benefit ratio, as FMT is a highly efficacious treatment for rCDI and can represent a life-saving treatment for affected patients.

The recurrence of CDI may lead to clinical progression of the disease. ${ }^{27}$ Therefore, the recurrence of CDI is more likely to appear as a severe disease rather than a first episode and is associated with a higher mortality. ${ }^{28} 29$ There are several studies that have shown a good efficacy of FMT for severe CDI using a variety of FMT protocols. ${ }^{2}$ 30-32

Based on this evidence, the panel encourages the use of FMT in clinical practice for the treatment of rCDI. This may also help decrease the associated economic burden on healthcare system, according to recent cost-effectiveness analysis. ${ }^{10} 11$

\section{FMT for refractory CDI}

Statement: FMT can be considered as a treatment option for refractory CDI.

Quality of evidence: low.

Strength of recommendation: strong.

Comment: A recent systematic review found seven nonrandomised observational studies in which FMT was used to treat patients with refractory CDI. ${ }^{6}$ None of them compared FMT with other therapies, and efficacy rates varied widely among studies (0\%-100\%; overall resolution rate, 55\%). In a recent double-bind RCT aimed at comparing frozen FMT with fresh FMT for the treatment of rCDI, a small portion of patients with refractory CDI were successfully treated both with frozen (per-protocol, 100\%) and fresh faeces (per-protocol, $67 \%)^{3}$

Moreover, refractory CDI often arises as a severe disease, ${ }^{27}$ which may be a life-threatening condition. As FMT appears to be an effective treatment of severe $\mathrm{CDI}^{2}{ }^{2}{ }^{30-32}$ it can also be considered an effective therapeutic option in CDI that is unresponsive to the usual antimicrobial treatment. However, further studies will need to consolidate this evidence.

FMT for the first episode of CDI

Statement: There is insufficient evidence to recommend FMT as a treatment for the first episode of CDI.

Quality of evidence: low.

Strength of recommendation: weak.
Comment: A recent systematic review found that FMT was used to treat an initial episode of CDI in a very small $(n=7)$ number of patients, with discrepant results. ${ }^{6}$ These data come from two small case series of patients with refractory or severe first episode of CDI. ${ }^{33}$ In addition, a preliminary report showed that treatment with FMT after the first episode of CDI can decrease mortality in patients infected with the 027 strain in comparison to FMT performed after recurrence. ${ }^{29}$ However, additional studies will need to establish if FMT is more costeffective than antibiotics for the treatment of the first episode of CDI.

\section{Other indications}

The experts panel took into account other clinical indications for a possible use of FMT in the clinical practice, such as IBD, IBS, metabolic disorders, paediatrics, but for none of them emerged an evidence-based recommendation to use FMT except that in a context of research (see online supplementary 1).

\section{Donor selection}

Key issue: collection of medical history

\section{General recommendations}

Statement: Potential donors for FMT have to undergo, at the beginning of the selection process, a medical interview to exclude history and risk factors (see box 1).

Quality of evidence: low.

Strength of recommendation: strong.

Comment: The main objective of donor selection is to reduce and prevent any adverse event related to the infused faecal material. Therefore, the panel recommends general exclusion criteria for donor selection before performing FMT, regardless of indications. As a first step, all potential donors should undergo a general questionnaire (preferably written) focused on their medical history and lifestyle habits to identify the risk factors. This is extremely relevant to exclude issues not detectable through blood and stool testing. To reduce the risk of donor comorbidities, individuals aged $<60$ years should be preferred. However, this indication cannot be mandatory in order not to foreclose the use of intimate healthy partners merely because of age. The panel chose the exclusion criteria shown in box 1 because they are the requirements of the European Commission for the selection of allogenic living donors of human tissue transplants. ${ }^{35}$ In addition, further exclusion criteria, especially those related to the presence of GI disorders, or drugs which may impair microbiota, were considered, based on available evidence. Indeed, the use of these or similar exclusion criteria assured, as shown in several RCTs, large case series and systematic review, ${ }^{1-3} 57$ 14-16 36 the occurrence of no or few adverse events related to the infusion of donors' material, as also stated in the French National Guidelines of FMT for the treatment of rCDI. ${ }^{22}$

Statement: Screened donors have to undergo a further interview on the same day of the donation, in order to check any recently onset potentially harmful issue (see box 2).

Quality of evidence: low.

Strength of recommendation: strong.

Comment: To ensure the safest possible procedure, the panel recommends a further check of recent history and risk factors of selected and laboratory screened donors on the same day of donation. The above recommendation is supported by excellent safety data from several RCTs, ${ }^{1} 21415$ and is also reported in the French National Guidelines of FMT for rCDI. ${ }^{22}$ 


\section{Box 1 Key issues to select potential donors at the preliminary interview}

INFECTIOUS DISEASES

- History of, or known exposure to, HIV, HBV or HCV, syphilis, human T-lymphotropic virus I and II, malaria, trypanosomiasis, tuberculosis

- Known systemic infection not controlled at the time of donation

- Use of illegal drugs

- Risky sexual behaviour (anonymous sexual contacts; sexual contacts with prostitutes, drug addicts, individuals with HIV, viral hepatitis, syphilis; work as prostitute; history of sexually transmittable disease)

- Previous reception of tissue/organ transplant

- Previous (<12 months) reception of blood products

- Recent ( $<6$ months) needle stick accident

- Recent ( $<6$ months) body tattoo, piercing, earring, acupuncture

- Recent medical treatment in poorly hygienic conditions

- Risk of transmission of diseases caused by prions

- Recent parasitosis or infection from rotavirus, Giardia lamblia and other microbes with GI involvement

- Recent ( $<6$ months) travel in tropical countries, countries at high risk of communicable diseases or traveller's diarrhoea

- Recent (<6 months) history of vaccination with a live attenuated virus, if there is a possible risk of transmission

- Healthcare workers (to exclude the risk of transmission of multidrug-resistant organisms)

- Individual working with animals (to exclude the risk of transmission of zoonotic infections)

\section{GI, METABOLIC AND NEUROLOGICAL DISORDERS}

- History of IBS, IBD, functional chronic constipation, coeliac disease, other chronic GI disorders

- History of chronic, systemic autoimmune disorders with GI involvement

- History of, or high risk for, Gl cancer or polyposis

- Recent appearance of diarrhoea, hematochezia

- History of neurological/neurodegenerative disorders

- History of psychiatric conditions

- Overweight and obesity (body mass index $>25$ )

\section{DRUGS THAT CAN IMPAIR GUT MICROBIOTA COMPOSITION}

- Recent (<3 months) exposure to antibiotics, immunosuppressants, chemotherapy

- Chronic therapy with proton pump inhibitors

\section{Box 2 Issues to address on the same day of donation to} check any recently onset of harmful events

- Newly appeared GI signs and symptoms, for example, diarrhoea, nausea, vomiting, abdominal pain, jaundice

- Newly appeared illness or general signs as fever, throat pain, swollen lymph nodes

- Use of antibiotics or other drugs that may impair gut microbiota, new sexual partners or travels abroad since the last screening

- Recent ingestion of a substance that may result harmful for the recipients

- Travel in tropical areas-contact with human blood (sting, wound, showing, piercings, tattoos)—sexual high-risk behaviour

- Diarrhoea (more than three loose or liquid stools per day) among members of the entourage (including children) within 4 weeks of donation

An alternative way, without exploiting the questionnaire, is to collect donor stools just after the results of the exams, and freeze and store them, if the centre has expertise in managing frozen material and has the availability of authorised stool bank facilities.
Recommendations for specific situations

See online supplementary 1.

Key issue: testing for donor selection

\section{General recommendations}

Statement: Suitable donors for FMT should undergo both blood and stool testing at most 4 weeks before donation (see box 3 ). If there are no changes in donor's health and specific circumstances, testing may be repeated up to 8 weeks.

Quality of evidence: low.

Strength of recommendation: strong.

Comment: The primary purpose of donor testing is to check the donor for infectious diseases potentially transmittable to the recipient. The selected blood and stool exams were shown to achieve an excellent safety profile in several RCTs of FMT. $^{1-3} 7$ 14-16 36 Some testing should be mandatory, with others as options, according to geographical areas (eg, human T-lymphotropic virus types I and II antibodies, or Strongyloides stercoralis), clinical conditions of recipients (eg, cytomegalovirus IgG, viral capsid antigen IgG, bacterial culture for Vibrio cholera and Listeria monocytogenes, antigens and/or acid fast-staining for Isospora and Microsporidia in the case of immunosuppressed recipients) or medical history of donors (eg, calprotectin). Although for some potential pathogen (such as human T-lymphotropic virus) there is no knowledge on transmission via FMT, the panel has expanded the boundaries of screening tests in order to ensure maximum safety profile to patients. Part 


\title{
Box 3 Blood and stool testing to check donors for any potentially transmittable disease
}

\author{
GENERAL BLOOD TESTING \\ - Cytomegalovirus \\ - Epstein-Barr virus \\ - Hepatitis A \\ - HBV \\ - $\mathrm{HCV}$ \\ - Hepatitis E virus \\ - Syphilis \\ - HIV-1 and HIV-2 \\ - Entamoeba histolytica \\ - Complete blood cell count with differential \\ - C-reactive protein and erythrocyte sedimentation rate \\ - Albumin \\ - Creatinine and electrolytes \\ - Aminotransferases, bilirubin, gamma-glutamyltransferase, alkaline phosphatase
}

BLOOD TESTING IN SPECIFIC SITUATIONS

- Human T-lymphotropic virus types I and II antibodies

- Strongyloides stercoralis

\section{GENERAL STOOL TESTING}

- Detection of Clostridium difficile

- Detection of enteric pathogens, including Salmonella, Shigella

- Campylobacter, Escherichia coli 0157 H7, Yersinia, vancomycin-resistant enterococci, methicillin-resistant Staphylococcus aureus, Gram-negative multidrug-resistant bacteria

- Norovirus

- Antigens and/or acid fast staining for Giardia lamblia and Criptosporidium parvum

- Protozoa (including Blastocystis hominis) and helminths

- Faecal occult blood testing

\section{STOOL TESTING IN SPECIFIC SITUATIONS}

- Detection of Vibrio cholera and Listeria monocytogenes

- Antigens and/or acid fast staining for Isospora and Microsporidia

- Calprotectin

- Helicobacter pylori faecal antigen

- Rotavirus

of blood testing, moreover, is required by the European Commission for the selection of allogenic living donors of human tissue transplants and by the French National Guidelines of FMT for rCDI. ${ }^{20} 35$

Most of the complications reported in the literature are principally related to the route of administration of faecal infusion and not to transmission of infection. However, there is poor evidence suggesting a precise deadline of donor testing before donation.

\section{Specific situations}

See online supplementary 1.

Key issue: choice of donors

Statement: Related or unrelated donors can be selected when FMT is performed to treat CDI. For other indications, the choice may be driven by specific needs.

Quality of evidence: moderate.

Strength of recommendation: strong.

Comment: According to the available evidence, there is no significant difference between patient-selected and anonymous healthy donors in terms of FMT outcomes, at least for rCDI, as shown by a meta-analysis. ${ }^{4}$ The use of both related and unrelated donors for FMT provided excellent rates of rCDI resolution in several RCTs and large case series. ${ }^{1} 23637$ Nevertheless, randomised studies comparing directly these categories of donors are still lacking. The use of unrelated healthy donors may be useful in FMT centres, with large volume of patients, to satisfy FMT requests. The availability of a stool bank in those centres is of utmost importance. Choice of related or unrelated donors might be driven by specific needs (eg, specific FMT indications, such as when there is a potential advantage of choosing unrelated donor to treat conditions with a pathogenic genetic basis; or in particular research designs). Finally, the advantage of excluding either related (eg, for IBD) or unrelated (eg, for paediatrics) donors still needs additional research to be addressed.

\section{Preparation of faecal material}

Key issue: stool handling and fresh faeces preparation

Statement: A minimum set of general steps has to be followed for the preparation of fresh faeces (see box 4).

Quality of evidence: moderate.

Strength of recommendation: strong.

Comment: The general steps (box 4) recommended by this statement are based on what has been described, but never 


\section{Box 4 Minimum general steps to follow for the preparation of fresh and frozen faecal material}

FRESH FAECAL MATERIAL

- Fresh stool should be used within 6 hours after defecation

- To protect anaerobic bacteria, the storage and preparation should be as brief as possible

- Until further processing, the stool sample can be stored at ambient temperature $\left(20^{\circ} \mathrm{C}-30^{\circ} \mathrm{C}\right)$

- Anaerobic storage and processing should be applied if possible

- A minimum amount of $30 \mathrm{~g}$ of faeces should be used

- Faecal material should be suspended in saline using a blender or manual effort and sieved in order to avoid the clogging of infusion syringes and tubes

- A dedicated space, disinfected using measures that are effective against sporulating bacteria, should be used

- Protective gloves and facial masks should be used during preparation

\section{FROZEN FAECAL MATERIAL}

- At least $30 \mathrm{~g}$ of donor faeces and $150 \mathrm{~mL}$ of saline solution should be used

- Before freezing, glycerol should be added up to a final concentration of $10 \%$

- The final suspension should be clearly labelled and traceable, and stored at $-80^{\circ} \mathrm{C}$

- On the day of faecal infusion, faecal suspension should be thawed in a warm $\left(37^{\circ} \mathrm{C}\right)$ water bath and infused within 6 hours from thawing

- After thawing, saline solution can be added to obtain a desired suspension volume

- Repetitive thawing and freezing should be avoided

rigorously tested. There are no reported studies comparing different preparation protocols of fresh faecal material, but the protocols used in different studies are comparable and allow good/moderate evidence of suitable protocols of fresh faecal preparation for FMT treatment of rCDI. ${ }^{1-3} 738$ The treatment efficacy with all considered protocols has been over $80 \%$. When reported, fresh faeces have been used for transplant within 6 hours after evacuation or on the same day. ${ }^{1-3} 738$ Both anaerobically and aerobically prepared samples are efficient in treatment of rCDI. ${ }^{1-3} 738$ This could be due to the fact that a considerable part of the bacterial genera of healthy microbiota produce resilient spores allowing interindividual transfer of at least a proportion of oxygen-sensitive intestinal bacteria. ${ }^{39}$ Given that these spore-forming bacteria typically represent about one-third of gut bacteria, ${ }^{39}$ and that disorders associated with microbiota alteration, such as IBS or IBD, are typically defined by lower abundance of anaerobic bacteria, ${ }^{40} 41$ it is rational to expect that the anaerobic processing of samples would be relevant for FMT success in the treatment of these disorders.

The amount of used faecal material varies from study to study, with most studies using $50 \mathrm{~g}$ of faeces ${ }^{42}$; nevertheless, recently, even $30 \mathrm{~g}$ of faecal material were shown to be sufficient for successful FMT. ${ }^{78}{ }^{43}$ However, the panel recognises that stool weight is a highly imperfect measure of microbiota quantity and acknowledge that there is wide variability in microbial content in stool between individuals and even different donations.

Moreover, the panel recommends faecal material to be diluted with sterile saline solution $(0.9 \%)$ with three to five times larger volume of solvent (eg, $30 \mathrm{~g}$ of faeces to be diluted in $150 \mathrm{~mL}$ of saline). In some studies, water has been successfully used as a solvent of faecal material (eg, Satokari et al). ${ }^{78}$ Although it has been reported that the preference should be given to saline as this solvent enables better preservation of microbes, ${ }^{44}$ conclusive comparative studies are missing. After homogenisation, the solids should be strained using gauze, ${ }^{3}$ tea strainer or similar device ${ }^{37}$ and suspension should be poured into a sterile container.
Key issue: preparation and defrosting of frozen faecal material Statement: Frozen faecal material can be used for FMT. A minimum set of general steps has to be followed for the preparation of frozen material (see box 4).

Quality of evidence: moderate.

Strength of recommendation: strong.

Comment: The FMT approach based on frozen faeces is essential for the development of a stool bank and is the optimal way to standardise the FMT process and to allow the availability of stool on demand, without delays associated with new screening. Comparative RCTs have shown similar efficiency of FMT performed with fresh and frozen faecal samples for the treatment of rCDI. ${ }^{3}{ }^{38}$ Similar to fresh samples, preparation of frozen faecal suspensions under normal air or under oxygen-free atmosphere yielded similar resolution rate. ${ }^{3} 3845$ For indications other than rCDI, anaerobic preparation might be more relevant, as indicated by higher resolution rates achieved with anerobically prepared samples when compared with aerobically prepared faecal suspensions. ${ }^{314}$ The panel recommends to use at least $30 \mathrm{~g}$ of donor faeces and $150 \mathrm{~mL}$ of saline solution. Before freezing, glycerol should be added up to a final concentration of $10 \% .^{38}$ Glycerol protects microbial cells from damage induced by freezing. ${ }^{46}$ On the contrary, the use of ethanol is still controversial. Despite encouraging phase I findings, ${ }^{47}$ ethanol-treated mix of sporulating bacteria failed to treat $\mathrm{rCDI}$ in a phase II study. ${ }^{48}$ It is plausible that ethanol, beyond killing pathogens, can also severely alter the composition of commensal microbiota, possibly eliminating critical elements such as bacteriophages, fungi and non-sporulating bacterial components.

The final suspension should be clearly labelled and traceable (using codes similar as for blood and tissue transplantation) and stored at $-80^{\circ} \mathrm{C}$. Storing at $-20^{\circ} \mathrm{C}$ might be feasible, ${ }^{3}$ but should be avoided since it allows some enzymes to be active, which can lead to degradation of sensitive microbial populations (eg, Bacteroidetes)..$^{49}$ Nevertheless, further studies are needed to show the optimal storage temperature of the frozen samples.

On the day of faecal infusion, faecal suspension should be thawed in a warm $\left(37^{\circ} \mathrm{C}\right)$ water bath. After thawing, saline solution could be added to obtain a desired suspension volume. The 
thawed faecal material should be infused within 6 hours after defrosting. Since microbial cells are sensitive after defrosting, ${ }^{50}$ repetitive thawing and freezing should be avoided and frozen samples should be prepared in doses needed for each infusion procedure.

Key issue: microbiota analysis of donors and recipients

See online supplementary 1.

\section{Clinical management and faecal delivery \\ Key issue: recipient preparation}

\section{Antibiotics}

Statement: Patients with rCDI should be treated with vancomycin or fidaxomicin at least for 3 days before FMT. Antibiotics should be stopped 12-48 hours before faecal infusion.

Quality of evidence: moderate.

Strength of recommendation: strong.

Comment: In patients with rCDI preinfusion use of metronidazole, vancomycin or fidaxomicin should be performed for at least 3 days, ${ }^{1-3} 17$ 20-22 in order to repress the abundance of intestinal C. difficile. However, antibiotics should be stopped 12-48 hours before stool application.

In emergencies, if frozen donor samples are immediately available, bridging therapy with antibiotics can be dispensed.

In indications other than $\mathrm{rCDI}$, for example, IBD, there are no high quality data to support any recommendation, even though antibiotic pretreatment could potentially increase clinical response. ${ }^{51}$

\section{Bowel lavage}

Statement: Recipients should be prepared with bowel lavage by polyethylene glycol before procedure when FMT is performed by upper route or by colonoscopy.

Quality of evidence: low.

Strength of recommendation: weak.

Comment: For delivery by colonoscopy, recipients receive a conventional colon lavage routinely given prior to colonoscopic examination. ${ }^{2} 172021$ As bowel lavage is able to reduce the abundance of C. difficile, it is also reasonable to suggest it also when FMT is performed via upper GI tract. ${ }^{1} 25253$ However, there is some report showing high success rate of FMT performed via upper GI tract and without prior bowel lavage. ${ }^{54}$ For delivery via enema, no preparation instructions have been developed. In particular, with a large RCT to treat rCDI by FMT, Lee et al obtained high cure rates using enema faecal delivery without colon lavage prior the procedure. ${ }^{3}$

Key issue: routes of faecal delivery

\section{FMT via colonoscopy}

Statement: When possible, donor stools should be infused into the right colon via the working channel of the colonoscope. In cases of severe colitis, faecal suspension can be disposed in the left colon for safety reasons.

Quality of evidence: high.

Strength of recommendation: strong.

Comment: Different routes of faecal delivery have been reported. Many systematic reviews and meta-analyses reported that colonoscopy achieved higher resolution rates of rCDI and similar safety profile than other routes of delivery. ${ }^{4-6}$ Depending on the general condition of the recipient, donor stool can be applied to the right or to the left colon. ${ }^{2} 7263155$ It does not seem to be very essential to dispose the suspension in multiple, smaller portions as it will distribute by gut peristalsis anyway. An amount of $200-500 \mathrm{~mL}$ of faecal suspension obtained from
20 to $100 \mathrm{~g}$ faeces can be delivered safely to the colon in contrast to other sites. ${ }^{2} 26374556$

\section{FMT via enema}

Statement: FMT can be applied by enema. Patients should be instructed to hold the infused material for at least $30 \mathrm{~min}$ and to remain supine to minimise the urge to defecate. The procedure could be repeated. ${ }^{3}$

Quality of evidence: low.

Strength of recommendation: strong.

Comment: Enema as route of administration have been reported in rCDI, in paediatric patients as well as in critical ill patients when colonoscopy was contraindicated. ${ }^{3-5}$ In other indications than rCDI, some study protocols implicate repeated enemas to increase clinical success. ${ }^{15}$ The enema route of administration may offer some advantages, as it is widely available and does not require costly devices. It is less invasive than other routes.

\section{FMT via upper GI tract}

Statement: FMT can be performed via upper GI tract. The faecal suspension can be delivered through the working channel of a gastroscope, or through nasogastric, nasojejunal or gastrostomy tube. Patients must be kept in a $45^{\circ}$ upright position for 4 hours after infusion in order to prevent aspiration.

Quality of evidence: high.

Strength of recommendation: strong.

Comment: When applied to the upper GI tract, total volumes of inserted bacterial suspension are much lower, about 25$50 \mathrm{~mL}$ compared with lower GI tract application. ${ }^{57}$ Volumes up to $500 \mathrm{~mL}$ have been placed via nasojejunal tubes over a longer period $(2-3 \mathrm{~min} / 50 \mathrm{~mL}){ }^{1}$

The peri-interventional use of prokinetics and proton pump inhibitors (PPIs) has been reported in case of upper GI delivery. ${ }^{2058} 59$ However, their beneficial use has not been proven yet.

Due to side effects and gut microbiota modifying effects of prokinetics and PPIs, their use should be considered cautiously. $^{60} 61$

Protocols using capsules containing faecal preparations for oral administration are up to now under investigation, with mixed results. ${ }^{62-64}$ This approach could expand the availability of FMT therapy in terms of accessibility and patient desirability. However, conclusive results are lacking, as comparative trials have not been performed yet.

Key issue: safety considerations

Statement: FMT appears to be safe even in immunocompromised and critically ill patients regardless the route of delivery. In case of critically ill patients, faecal infusion by enema(s) should be preferred.

Quality of evidence: low.

Strength of recommendation: strong.

Comment: FMT has been performed in elderly, ${ }^{65}$ immunocompromised $^{6667}$ as well as in critically ill patients, via upper and lower GI tract. However, the rate of serious adverse events in recipients who received FMT via upper GI tract seems to be higher. Aspiration pneumonia due to nausea and vomiting by a nasogastric or nasojejunal tube has been reported. ${ }^{1}{ }^{5768}$ Fever has been observed both after via-upper GI and (less frequently) after via-colonoscopy infusion. ${ }^{38} 55586768$ In the study by Moayyedi et al, ${ }^{15}$ rectal abscesses after via-enemas infusion of faeces or placebo were reported in patients with UC. However, perianal disease in UC is also known to occur regardless of FMT. $^{69}$ 
Key issue: repeated faecal infusion

Statement: Faecal infusion can be repeated in case of treatment failure or clinical recurrence of CDI.

Quality of evidence: high.

Strength of recommendation: strong.

Comment: In severe CDI infection, especially in colitis with endoscopic evidence of pseudomembranes, repetition of faecal infusion had been necessary to induce clinical remission. $^{2}$ 30-32 70-72 Success of FMT may also depend on the amount of delivered faecal suspension, which can be increased by multiple infusions. ${ }^{3}$ Factors negatively influencing the outcome of FMT might be low volume of donor stool, severe colitis and/or ongoing antibiotic treatment and route of delivery, together with frequency of CDI-related hospitalisation. ${ }^{72-74}$ Stool from another donor should be considered in case of failed clinical resolution despite multiple infusions.

In other indications than rCDI, such as IBD, repeated FMTs have been reported to increase the success rate. ${ }^{14} 15517576$ Nevertheless, in many other potential indications than rCDI proven standard procedures are lacking.

Key issue: monitoring of patients (adverse events and efficacy outcomes)

\section{Short-term monitoring of patients for adverse events}

Statement: Recipients should be monitored for the occurrence of possible acute complications related to the procedure.

\section{Quality of evidence: low.}

Strength of recommendation: weak.

Comment: Infection control practices of patients with rCDI should be performed as recommended by international guidelines, ${ }^{12}{ }^{13}$ according to disease severity and comorbidities. The need for hospitalisation of patients with other underlying diseases depends on the diagnosis and clinical condition. When repeated faecal infusions are necessary, provided that the patient's condition is good, further applications can be performed in an outpatient setting. ${ }^{15}$ The duration of the observation period has not been defined yet, as it depends on the route of delivery, the underlying diseases and the general condition of the patient. ${ }^{22}$

Most common short term adverse events after FMT due to CDI have been described as diarrhoea, abdominal cramps, belching, constipation, fever as well as Gram-negative bacteraemia and perforation. ${ }^{1} 4265$ In IBD, diarrhoea, abdominal bloating and cramping, fever and deterioration of disease have been reported. ${ }^{5158} 7778$ Mortality has been reported as an outcome due to aspiration during sedation after FMT via colonoscopy. ${ }^{79}$ Another patient died after regurgitation of faecal material infused into the duodenum under general anaesthesia. ${ }^{67}$ After delivering donor stool via a pre-existing nasogastric tube, another patient developed septic shock and toxic megacolon and died after colonic resection. ${ }^{80}$ Finally, a patient had a sever septic shock due to the faecal aspiration following endoscopic peroral jejunal FMT. ${ }^{54}$

Long-term monitoring of patients for adverse events

Statement: Periodicity and length of follow-up for long-term adverse events are not determined. Follow-up should include clinical and analytical data.

\section{Ouality of evidence: low.}

Strength of recommendation: weak.

Comment: To date, there are not enough long-term follow-up data to estimate potential FMT-related diseases. A single case of weight gain has been reported, as well as the development of peripheral neuropathy, Sjögren's disease, idiopathic thrombocytopenic purpura and rheumatoid arthritis. ${ }^{37} 81$ There are other anecdotal reports about improvement of non CDI-related diseases after FMT as IBS, chronic constipation, antibiotic-induced noninfectious colitis as well as extraintestinal diseases as Parkinson's disease, multiple sclerosis and idiopathic thrombocytopenic purpura. $^{82}{ }^{83}$ However, causality to FMT remains unclear. The transmission of malignant, autoimmune, metabolic or neuropsychiatric diseases have been discussed and at least partially shown in animal models. ${ }^{84}$ Patients with CDI are known to be at high risk of postinfectious IBS. ${ }^{85}$ However, the role of FMT in this context has to be elucidated.

The implementation of registry data collections can effectively deal the issue of long-term monitoring of patients for adverse events.

\section{Monitoring of patients for efficacy outcomes}

Statement: Patients receiving FMT for CDI should be followed up for at least 8 weeks.

Quality of evidence: low.

Strength of recommendation: strong.

Comment: Treatment response implies clinical improvement, ${ }^{13}$ as reduction of stool frequency and improvement of stool consistency, as well as amelioration of other parameters of disease severity as laboratory parameters, radiological and/or endoscopic findings. Repetition of $C$. difficile testing in stool samples is generally not recommended, as toxins might stay positive for weeks. In clinical practice, it is not rational to distinguish between recurrence and relapse of CDI.

\section{Basic requirements for implementing an FMT centre}

Key issue: clinical requirements and facilities

Statement: Development of referral FMT centres for the treatment of CDI in clinical practice is encouraged. Centres should be implemented in hospitals with appropriate expertise and facilities.

Quality of evidence: moderate.

Strength of recommendation: strong.

Comment: CDI is the most common cause of diarrhoea in inpatients, and is increasing in incidence, morbidity, mortality and likelihood to recur. ${ }^{8}$ On the other hand, CDI not responding to standard therapy is a complex disease requiring clinical expertise. FMT achieved significant advantage over standard antibiotic therapy for the management of rCDI in RCTs, ${ }^{12}$ and is clearly recognised as a reliable and cost-effective treatment for this burdensome disease. ${ }^{6} 911$ Therefore, the dissemination of the FMT procedure and the establishment of FMT services into clinical setting could be useful practices to reduce the healthcare burden of $\mathrm{rCDI},{ }^{43}$ as advocated by physicians interested into gastroenterology and infectious diseases. ${ }^{18} 1986-89$ The development of an FMT centre service could fill the therapeutic gap for the management of the life-threatening rCDI by ensuring the optimal standardisation of the FMT process.

Statement: In order to establish an FMT centre, actively involved members are encouraged to undergo a specific training on FMT processes.

Quality of evidence: low.

Strength of recommendation: strong.

Comment: As with other technical procedures, a specific training period is required before performing FMT. Therefore, physicians who are going to implement FMT in their centre are encouraged to undergo a prior training. Essentially, training on FMT is composed of three parts: clinical training, which includes donor and patient selection, and patient management after FMT; delivery training, which includes the learning of 
different routes of delivery (eg, enema, colonoscopy, nasoduodenal/nasojejunal tube, etc) and microbiological training (preparation of fresh and frozen faecal material).

Statement: The assemblage of a multidisciplinary team, including gastroenterologists, microbiologists and infectious disease physicians, is encouraged to build an FMT centre.

Quality of evidence: low.

Strength of recommendation: strong.

Comment: There are no published data supporting this statement, and evidence comes from opinions of the panel, based on clinical experience, descriptive case studies or reports of expert committees. Nevertheless, the panel strongly believes that the development of a multidisciplinary team, composed of gastroenterologists, microbiologists and infectious disease physicians, will be extremely useful to build an FMT referral centre, because it would gather different and complementary expertises.

Statement: The availability of several facilities (including endoscopy service, clinical ward and outpatient clinic) is mandatory to implement an FMT centre. FMT can be performed within either inpatient or outpatient setting.

Quality of evidence: low.

Strength of recommendation: strong.

Comment: There are no published data supporting this statement and evidence comes from opinions of the expert panel, based on clinical experience, descriptive case studies or reports of expert committees. Provided that the patient's condition is stable, faecal infusion can be performed in an outpatient setting. ${ }^{20}$ The panel strongly believes that several facilities are needed to build an FMT referral centre, to offer FMT to both patients with mild and severe CDI, to manage potential adverse events related either to the procedure or to the infused material and to easily follow-up patients after FMT.

Statement: A clinical governance dealing with administrative issues of FMT is recommended to develop an FMT centre.

Quality of evidence: low.

Strength of recommendation: strong.

Comment: There are no published data supporting this statement and evidence comes from opinions of the expert panel, based on clinical experience, descriptive case studies or reports of expert committees. The panel believes that the implementation of a clinical governance within the FMT centre would be extremely useful to deal with administrative issues related to FMT (eg, reimbursements, authorisations), and to overcome all bureaucratical and organisational barriers that may prevent the development and the work of the centre itself.

\section{Key issue: microbiological requirements and facilities}

Statement: FMT centres need to have an access or be part of the facility that allows safe processing of human samples (biosafety level 2) including aliquoting, storage and preparation of faeces. Stool banking is encouraged.

Quality of evidence: low.

Strength of recommendation: strong.

Comment: There are no evidence-based data supporting the recommendation of high-quality microbiological requirements for building an FMT Centre. Current recommendations include expert opinions from the expert panel and existing general recommendation including guidelines for safe work practice..$^{90-92}$

One of the key functions of the FMT centre is the management of blood and faecal samples from either donor or recipients. C. difficile is a pathogen with a biosafety level 2 (cabinet with high-efficiency particulate air filter) and according, safety requirements and recommendation need to be implemented. ${ }^{90}$ Material processing (fresh faeces, bank of frozen and stored faeces) and safety precautions have to adhere to basic principles for safe preparation of human material, including: rigorous protocols in securing the materials; maintenance of standard operating procedures (SOPs) for the processing; use of certified laboratory testing; definition of quality control tests and standards for the release of the final product.

There are numerous advantages associated with the implementation of stool banks (such as the OpenBiome initiative in the USA). A frozen stool bank allows faecal donors to be recruited and thoroughly screened ahead of time, in a methodical manner, without time pressure and with the potential advantage to reduce the costs associated with donor screening as one donor can serve for multiple FMT donations. Furthermore, the accumulation of expertise and the standardisation of FMT processing ultimately allow optimising the quality control, a greater potential for rigour of testing and the coordination of research activities/outcomes monitoring. Finally, stool banks may improve the accessibility of FMT to centres that otherwise would be unable to provide the service due to inadequate resources to conduct donor recruitment/screening and FMT processing.

Statement: FMT procedure and donor screening documentation should be stored for at least 10 years (unless local requirements consider longer retention period) in order to archive donor material in case of future adverse events.

Quality of evidence: low.

Strength of recommendation: strong.

Comment: Management of the records related to the FMT procedure should be regulated by local health organisation. FMT procedure and donors' and recipients' records should be stored for at least 10 years. This may differ according to local requirements, and longer storage time could be needed. The records of the FMT centre will provide access to the long-term safety data.

Samples of donor faeces before the processing and before the administration to recipients should be stored for a possible microbiological evaluation and qualitative and quantitative characterisation due to safety reasons. The frozen samples should be stored in adequate facilities (or, when specifically regulated, by authorised facilities) and should be clearly labelled with the code of the donor and the date of donation.

Key issue: regulatory requirements

Statement: Appropriate FMT registries should be implemented, in order to collect data concerning indications, procedure, effectiveness and safety profiles.

Quality of evidence: low.

Strength of recommendation: strong.

Comment: There are no published data supporting this statement, and evidence comes from opinions of the expert panel, based on clinical experience or on reports of expert committees. Recently, the American Gastroenterological Association established a National Institutes of Health-funded registry to track patient outcomes associated with $\mathrm{FMT}^{93}$ with the ultimate objective to protect the well-being of patients and guide further scientific exploration in the field. Such a registry would serve as a source of short-term and long-term information about the clinical practice of FMT in the USA.

FMT has not undergone traditional regulatory approval process of pharmaceutical products with sequential testing leading to large phase III trials assessing efficacy and safety prior to clinical utilisation. Therefore, the panel believes that the creation of registries to be kept at the local (hospital) and/or at the regional, national or international competent authorities is relevant to collect data and could be useful to deal with outcomes and safety issues related to FMT. In order to trace causality of 
FMT and newly developed diseases, traceability by keeping appropriate registries would be a wise process to learn about potential long-term side effects.

Statement: Specific national rules for the classification of FMT should be followed to implement an FMT centre.

Quality of evidence: low.

Strength of recommendation: strong.

Comment: A commonly acknowledged regulatory classification for FMT has not been established yet in Europe. Several countries have introduced some national rules and others require to be compliant to the European directive 2004/23 on quality and safety of tissues and cells. ${ }^{94}$

As FMT falls under the category of 'Substance of Human Origin' like cells, tissues, milk, etc., the most important requirements should be followed, as for example an adequate facility.

Activities and responsibilities for processing and testing the raw material, for use of equipment, for preservation and storage and for release and distribution of FMT, should be described in SOPs.

\section{CONCLUSIONS}

This consensus indicates that the only clinical indication with sufficient evidence of benefit from the implementation of FMT in clinical practice is CDI (see online supplementary 2). The consensus panel strongly recommends the implementation of FMT centres for the treatment of CDI in adults.

CDI is the most common cause of infectious diarrhoea among hospitalised patients, and, despite advances in both drug treatment and infection control practices, complications rates (such as sepsis, toxic megacolon, death) continue to increase, mainly due to treatment failure and recurrence of infection. Therefore, CDI may represent a considerable therapeutic challenge for clinicians. The clinical burden of rCDI is intuitively tied to a commensurate economic burden, which is principally due to the increased duration of hospitalisation or re-admission and the management of complications.

On the other hand, although FMT has been shown to achieve excellent cure rates with few serious adverse events, physicians together with hospital organisations are not ready yet to offer this option of treatment to such patients because a series of technical, logistical and bureaucratic issues have hampered the development of an FMT capability at many hospitals. This mainly occurs because a clear global regulation is still lacking, particularly in Europe.

We believe that the results obtained by this evidence-based consensus conference are important because they reiterate the need to make use of the FMT for the treatment of CDI, establish technical guidelines of the procedure and mostly indicate basic requirements for an organisative model, which is essential to implement FMT in the clinical practice within an appropriate safety control and clinical governance (see online supplementary 2). At last, this can help to make a life-saving treatment option for patients widely available, with a profitable impact on the healthcare system.

Moreover, there is no strong evidence-based recommendation for the use of FMT in other clinical conditions, although interesting findings come from the application of FMT for the treatment of UC, ${ }^{15} \mathrm{MS},{ }^{16} \mathrm{IBS}^{95}$ and, more recently, graft-versus-host disease. ${ }^{96}$ Therefore, the experience resulting from an FMT approach to CDI could be translated in terms of scientific information, technical know-how and knowledge dissemination platforms to other research areas, with the ultimate goal of understanding the role that may be played by FMT, in the future, in other clinical conditions.

\section{Author affiliations}

1Department of Gastroenterological Area, "A. Gemelli" Hospital, Catholic University of the Sacred Heart, Rome, Italy

${ }^{2}$ Department of Internal Medicine I, Gastroenterology, Endocrinology \& Metabolism, Medical University, Innsbruck, Austria

${ }^{3}$ Faculty of Technology and Metallurgy, Department of Biochemical Engineering and

Biotechnology, University of Belgrade, Belgrade, Serbia

${ }^{4}$ Division of Gastroenterology and Hepatology, Department of Internal Medicine,

Medical University, Graz, Austria

${ }^{5}$ Faculty of Medicine, Immunobiology Research Program, Department of Bacteriology and Immunology, University of Helsinki, Helsinki, Finland

${ }^{6}$ Gastroenterology and Nutrition Department, AP-HP, French Group of Faecal

Microbiota Transplantation (GFTF), Saint-Antoine Hospital and UPMC Paris 06,

Paris, France

${ }^{7}$ Department of Clinic of Gastroenterology, University of Helsinki and Helsinki University Hospital, Helsinki, Finland

${ }^{8}$ Tissues and Cells Area, Italian National Transplant Center (CNT), Rome, Italy

${ }^{9}$ Department of Gastroenterology, St. Mark's Hospital, London, UK

${ }^{10}$ Department of Pediatrics, Pediatric Gastroenterology and Liver Unit, Sapienza

University, Rome, Italy

${ }^{11}$ Laboratory of Microbiology, "A. Gemelli" Hospital, Catholic University of the

Sacred Heart, Rome, Italy

${ }^{12}$ Department of Molecular and Clinical Medicine, Wallenberg Laboratory, University

of Gothenburg, Institute of Medicine, Sahlgrenska Academy, Gothenburg, Sweden

${ }^{13}$ Gastroenterology and Hepatology Service, Ramón y Cajal University Hospital,

Madrid, Spain

${ }^{14}$ Department of Gastroenterology, Hepatology and Infectious Diseases,

Otto-von-Guericke University Hospital, Magdeburg, Germany

${ }^{15}$ Department of Internal Medicine, Academic University Medical Center,

Amsterdam, The Netherlands

${ }^{16}$ Laboratory of Microbiology, Wageningen University, Wageningen, The Netherlands

${ }^{17}$ Department of Infectious Diseases, Helsinki University Central Hospital, Helsinki,

Finland

${ }^{18} \mathrm{Clinic}$ for Gastroenterology and Hepatology, University of Belgrade and School of Medicine, Clinical Center of Serbia, Belgrade, Serbia

${ }^{19}$ Department of Internal Medicine, VU University Medical Center, Amsterdam, The Netherlands

${ }^{20}$ Department of Internal Medicine and Clinical Nutrition, University of Gothenburg, Sahlgrenska Academy, Gothenburg, Sweden

\section{Twitter Follow Gianluca laniro @gianluca1aniro}

Contributors GC, GI and AG planned the meeting and established the main topics. All panel members were involved in developing the statements with supporting evidence and drafted the text of discussion relevant to their statements. GC wrote the initial draft of the manuscript. All panel faculties read and revised the manuscript for important intellectual content and approved the final manuscript.

Funding MN is supported by a ZONMW-VIDI grant 2013 (016.146.327) and CVON by a Young Talent grant 2012. RS is supported by Academy of Finland (258439).

Competing interests $\mathrm{MN}$ is in the scientific advisory board of Seres Therapeuticals and Caelus Health.

Provenance and peer review Not commissioned; externally peer reviewed.

Open Access This is an Open Access article distributed in accordance with the Creative Commons Attribution Non Commercial (CC BY-NC 4.0) license, which permits others to distribute, remix, adapt, build upon this work non-commercially, and license their derivative works on different terms, provided the original work is properly cited and the use is non-commercial. See: http://creativecommons.org/ licenses/by-nc/4.0/

\section{REFERENCES}

1 van Nood E, Vrieze A, Nieuwdorp M, et al. Duodenal infusion of donor feces for recurrent Clostridium difficile. N Engl J Med 2013;368:407-15.

2 Cammarota G, Masucci L, laniro G, et al. Randomised clinical trial: faecal microbiota transplantation by colonoscopy vs. vancomycin for the treatment of recurrent Clostridium difficile infection. Aliment Pharmacol Ther 2015;41:835-43.

3 Lee $\mathrm{CH}$, Steiner T, Petrof EO, et al. Frozen vs fresh fecal microbiota transplantation and clinical resolution of diarrhea in patients with recurrent Clostridium difficile infection: a randomized clinical trial. JAMA 2016;315:142-9.

4 Kassam Z, Lee $\mathrm{CH}$, Yuan Y, et al. Fecal microbiota transplantation for Clostridium difficile infection: systematic review and meta-analysis. Am J Gastroenterol 2013;108:500-8.

5 Cammarota G, laniro G, Gasbarrini A. Fecal microbiota transplantation for the treatment of Clostridium difficile infection: a systematic review. J Clin Gastroenterol 2014;48:693-702. 
6 Drekonja D, Reich J, Gezahegn S, et al. Fecal microbiota transplantation for Clostridium difficile infection: a systematic review. Ann Intern Med 2015;162:630-8.

7 Mattila E, Uusitalo-Seppälä R, Wuorela M, et al. Fecal transplantation, through colonoscopy, is effective therapy for recurrent Clostridium difficile infection. Gastroenterology 2012;142:490-6.

8 Lessa FC, Mu Y, Bamberg WM, et al. Burden of Clostridium difficile infection in the United States. N Engl J Med 2015;372:825-34.

9 Varier RU, Biltaji E, Smith KJ, et al. Cost-effectiveness analysis of fecal microbiota transplantation for recurrent C. difficile infection. Infect Control Hosp Epidemiol 2015;36:438-44

10 McGlone SM, Bailey RR, Zimmer SM, et al. The economic burden of Clostridium difficile. Clin Microbiol Infect 2012;18:282-9.

11 Waye A, Atkins K, Kao D. Cost averted with timely fecal microbiota transplantantion in the management of recurrent Clostridium difficile infection in Alberta, Canada. J Clin Gastroenterol 2016;50:747-53.

12 Surawicz CM, Brandt LJ, Binion DG, et al. Guidelines for diagnosis, treatment, and prevention of Clostridium difficile infections. Am J Gastroenterol 2013;108:478-98.

13 Debast SB, Bauer MP, Kuijper EJ, European Society of Clinical Microbiology and Infectious Diseases. European Society of Clinical Microbiology and Infectious Diseases: update of the treatment guidance document for Clostridium difficile infection. Clin Microbiol Infect 2014;20(Suppl 2):1-26.

14 Rossen NG, Fuentes $S$, van der Spek MJ, et al. Findings from a randomized controlled trial of fecal transplantation for patients with ulcerative colitis. Gastroenterology 2015;149:110-18.

15 Moayyedi P, Surette MG, Kim PT, et al. Fecal microbiota transplantation induces remission in patients with active ulcerative colitis in a randomized controlled trial. Gastroenterology 2015;149:102-9.

16 Vrieze A, Van Nood E, Holleman F, et al. Transfer of intestinal microbiota from lean donors increases insulin sensitivity in individuals with metabolic syndrome. Gastroenterology 2012;143:913-16.

17 Trubiano JA, Cheng AC, Korman TM, et al. Australasian Society of Infectious Diseases updated guidelines for the management of Clostridium difficile infection in adults and children in Australia and New Zealand. Intern Med J 2016;46:479-93.

18 Jiang ZD, Hoang LD, Lasco TM, et al. Physician attitudes toward the use of fecal transplantation for recurrent Clostridium difficile infection in a metropolitan area. Clin Infect Dis 2013;56:1059-60.

19 Zipursky JS, Sidorsky TI, Freedman CA, et al. Patient attitudes toward the use of fecal microbiota transplantation in the treatment of recurrent Clostridium difficile infection. Clin Infect Dis 2012;55:1652-8.

20 Bakken JS, Borody T, Brandt LJ, et al. Treating Clostridium difficile infection with fecal microbiota transplantation. Clin Gastroenterol Hepatol 2011;9:1044-9.

21 Kump PK, Krause R, Steininger C, et al. Recommendations for the use of faecal microbiota transplantation "stool transplantation": consensus of the Austrian Society of Gastroenterology and Hepatology (ÖGGH) in cooperation with the Austrian Society of Infectious Diseases and Tropical Medicine. $Z$ Gastroenterol 2014;52:1485-92.

22 Sokol H, Galperine T, Kapel N, et al. Faecal microbiota transplantation in recurrent Clostridium difficile infection: Recommendations from the French Group of Faecal Microbiota Transplantation. Dig Liver Dis 2016;48:242-7.

23 Guyatt GH, Oxman AD, Vist GE, et al. GRADE: an emerging consensus on rating quality of evidence and strength of recommendations. BMJ 2008;336:924-6.

24 Atkins D, Best D, Briss PA, et al. Grading quality of evidence and strength of recommendations. BMJ 2004;328:1490.

25 Hsu CC, Sandford BA. The Delphi technique: making sense of consensus. Pract Assess Res Eval 2007:12:1-6.

26 Kelly CR, Khorutz A, Staley C, et al. Effect of fecal microbiota transplantation on recurrence in multiply recurrent Clostridium difficile infection. Ann Intern Med 2016;165:609-16.

27 Surawicz CM, Alexander J. Treatment of refractory and recurrent Clostridium difficile infection. Nat Rev Gastroenterol Hepatol 2011;8:330-9.

28 Olsen MA, Yan Y, Reske KA, et al. Recurrent Clostridium difficile infection is associated with increased mortality. Clin Microbiol Infect 2015;21:164-70

29 Lagier JC, Delord M, Million M, et al. Dramatic reduction in Clostridium difficile ribotype 027 -associated mortality with early fecal transplantation by the nasogastric route: a preliminary report. Eur J Clin Microbiol Infect Dis 2015;34:1597-601.

30 Cammarota G, laniro G, Magalini S, et al. Decrease in surgery for Clostridium difficile infection after starting a program to transplant fecal microbiota. Ann Intern Med 2015;163:487-8.

31 Fischer M, Sipe BW, Rogers NA, et al. Faecal microbiota transplantation plus selected use of vancomycin for severe-complicated Clostridium difficile infection: description of a protocol with high success rate. Aliment Pharmacol Ther 2015;42:470-6.

32 Weingarden AR, Hamilton MJ, Sadowsky MJ, et al. Resolution of severe Clostridium difficile infection following sequential fecal microbiota transplantation. J Clin Gastroenterol 2013;47:735-7.

33 Lee $\mathrm{CH}$, Belanger JE, Kassam Z, et al. The outcome and long-term follow-up of 94 patients with recurrent and refractory Clostridium difficile infection using single to multiple fecal microbiota transplantation via retention enema. Eur J Clin Microbiol Infect Dis 2014;33:1425-8.
34 Eiseman B, Silen W, Bascom GS, et al. Fecal enema as an adjunct in the treatment of pseudomembranous enterocolitis. Surgery 1958;44:854-9.

35 Commission Directive 2006/17/EC of 8 February 2006 implementing Directive 2004 23/EC of the European Parliament and of the Council as regards certain technical requirements for the donation, procurement and testing of human tissues and cells.

36 Youngster I, Sauk J, Pindar C, et al. Fecal microbiota transplant for relapsing Clostridium difficile infection using a frozen inoculum from unrelated donors: a randomized, open-label, controlled pilot study. Clin Infect Dis 2014:58:1515-22.

37 Brandt LJ, Aroniadis OC, Mellow M, et al. Long-term follow-up of colonoscopic fecal microbiota transplant for recurrent Clostridium difficile infection. Am J Gastroenterol 2012;107:1079-87.

38 Satokari R, Mattila E, Kainulainen V, et al. Simple faecal preparation and efficacy of frozen inoculum in faecal microbiota transplantation for recurrent Clostridium difficile infection — an observational cohort study. Aliment Pharmacol Ther 2015;41:46-53.

39 Browne HP, Forster SC, Anonye BO, et al. Culturing of 'unculturable' human microbiota reveals novel taxa and extensive sporulation. Nature 2016;533:543-6.

40 Sokol H, Seksik P, Furet JP, et al. Low counts of Faecalibacterium prausnitzii in colitis microbiota. Inflamm Bowel Dis 2009;15:1183-9.

41 Rajilić-Stojanović M, Biagi E, Heilig HGHJ, et al. Global and deep molecular analysis of microbiota signatures in fecal samples from patients with irritable bowel syndrome. Gastroenterology 2011;141:1792-801.

42 Gough E, Shaikh H, Manges AR. Systematic review of intestinal microbiota transplantation (fecal bacteriotherapy) for recurrent Clostridium difficile infection. Clin Infect Dis 2011;53:994-1002.

43 Costello SP, Tucker EC, La Brooy J, et al. Establishing a fecal microbiota transplant service for the treatment of Clostridium difficile infection. Clin Infect Dis 2016;62:908-14.

44 Liao $\mathrm{CH}$, Shollenberger LM. Survivability and long-term preservation of bacteria in water and in phosphate-buffered saline. Lett Appl Microbiol 2003;37:45-50.

45 Hamilton MJ, Weingarden AR, Sadowsky MJ, et al. Standardized frozen preparation for transplantation of fecal microbiota for recurrent Clostridium difficile infection. Am I Gastroenterol 2013;107:761-7.

46 Fuller BJ. Cryoprotectants: the essential antifreezes to protect life in the frozen state. Cryo Letters 2004;25:375-88.

47 Khanna S, Pardi DS, Kelly CR, et al. A novel microbiome therapeutic increases gut microbial diversity and prevents recurrent Clostridium difficile infection. J Infect Dis 2016;214:173-81.

48 Seres Therapeutics Announces Interim Results from SER-109 Phase 2 ECOSPOR Study in Multiply Recurrent Clostridium Difficile Infection. http://ir.serestherapeutics.com/ phoenix.zhtml? $c=254006 \& p=$ irol-newsArticle\&ID=2190006 (accessed 6 Oct 2016).

49 Bahl MI, Bergström A, Licht TR. Freezing fecal samples prior to DNA extraction affects the Firmicutes to Bacteroidetes ratio determined by downstream quantitative PCR analysis. FEMS Microbiol Lett 2012;329:193-7.

50 Sleight SC, Wigginton NS, Lenski RE. Increased susceptibility to repeated freeze-thaw cycles in Escherichia coli following long-term evolution in a benign environment. BMC Evol Biol 2006;6:104.

51 Kump PK, Gröchenig HP, Spindelbock W, et al. Preliminary clinical results of repeatedly fecal microbiota transplantation (FMT) in chronic active ulcerative colitis [abstract]. United European Gastroenterol J 2013;1S:A57.

52 Gorkiewicz G, Thallinger GG, Trajanoski S, et al. Alterations in the colonic microbiota in response to osmotic diarrhea. PLoS One 2013;8:e55817.

53 Jalanka J, Salonen A, Salojärvi J, et al. Effects of bowel cleansing on the intestinal microbiota. Gut 2015;64:1562-8.

54 Link A, Lachmund T, Schulz C, et al. Endoscopic peroral jejunal fecal microbiota transplantation. Dig Liv Dis 2016;48:1336-9.

55 Kump PK, Gröchenig HP, Lackner $S$, et al. Alteration of intestinal dysbiosis by fecal microbiota transplantation does not induce remission in patients with chronic active ulcerative colitis. Inflamm Bowel Dis 2013;19:2155-65.

56 Kelly $C R$, de Leon L, Jasutkar N. Fecal microbiota transplantation for relapsing Clostridium difficile infection in 26 patients: methodology and results. I Clin Gastroenterol 2012;46:145-9.

57 Aas J, Gessert CE, Bakken JS. Recurrent clostridium difficile colitis: case series involving 18 patients treated with donor stool administered via a nasogastric tube. Clin Infect Dis 2003:36:580-5.

58 Angelberger S, Reinisch W, Makristathis A, et al. Temporal bacterial community dynamics vary among ulcerative colitis patients after fecal microbiota transplantation. Am J Gastroenterol 2013;108:1620-30.

59 Suskind DL, Singh N, Nielson $\mathrm{H}$, et al. Fecal microbial transplant via nasogastric tube for active pediatric ulcerative colitis. J Pediatr Gastroenterol Nutr 2015;60:27-9.

60 Imhann F, Bonder MJ, Vich Vila A, et al. Proton pump inhibitors affect the gut microbiome. Gut 2016:65:740-8

61 Freedberg DE, Toussaint NC, Chen SP, et al. Proton pump inhibitors alter specific taxa in the human gastrointestinal microbiome: a crossover trial. Gastroenterology 2015;149:883-5.

62 Hirsch BE, Saraiya N, Poeth K, et al. Effectiveness of fecal-derived microbiota transfer using orally administered capsules for recurrent Clostridium difficile infection. BMC Infect Dis 2015;17:191. 
63 Youngster I, Russell GH, Pindar C, et al. Oral, capsulized, frozen fecal microbiota transplantation for relapsing Clostridium difficile infection. JAMA 2014;5:1772-8.

64 http://ir.serestherapeutics.com/phoenix.zhtml?c=254006\&p=irol-newsArticle\&ID= 2190006

65 Agrawal M, Aroniadis OC, Brandt LJ, et al. The long-term efficacy and safety of fecal microbiota transplant for recurrent, severe, and complicated clostridium difficile infection in 146 elderly individuals. J Clin Gastroenterol 2016;50: 403-7.

66 Dao MC, Everard A, Aron-Wisnewsky J, et al. Akkermansia muciniphila and improved metabolic health during a dietary intervention in obesity: relationship with gut microbiome richness and ecology. Gut 2016;65:426-36.

67 Frank J, Högenauer C, Gröchenig HP, et al. Safety of fecal microbiota transplantation in patients with chronic colitis and immunosuppressive treatment [abstract]. J Crohns Colitis 2015;9:S245.

68 Baxter M, Ahmad T, Colville A, et al. Fatal aspiration pneumonia as a complication of fecal microbiota transplant. Clin Infect Dis 2015;61:136-7.

69 Zabana Y, Van Domselaar M, Garcia-Planella $E$, et al. Perianal disease in patients with ulcerative colitis: a case-control study. J Crohns Colitis 2011;5:338-41.

70 Gweon TG, Kim J, Lim CH, et al. Fecal microbiota transplantation using upper gastrointestinal tract for the treatment of refractory or severe complicated Clostridium difficile infection in elderly patients in poor medical condition: the first study in an Asian country. Gastroenterol Res Pract 2016;2016:2687605.

71 Aroniadis OC, Brandt LJ, Greenberg A, et al. Long-term follow-up study of fecal microbiota transplantation for severe and/or complicated Clostridium difficile infection: a multicenter experience. J Clin Gastroenterol 2016;50:398-402.

72 Fischer M, Kao D, Mehta SR, et al. Predictors of early failure after fecal microbiota transplantation for the therapy of Clostridium difficile infection: a multicenter study. Am J Gastroenterol 2016:111:1024-31.

73 Furuya-Kanamori L, Doi SA, Paterson DL, et al. Upper versus lower gastrointestinal delivery for transplantation of fecal microbiota in recurrent or refractory Clostridium difficile infection: a collaborative analysis of individual patient data from 14 studies J Clin Gastroenterol. Online Published First: 11 March 2016.

74 Meighani A, Hart BR, Mittal C, et al. Predictors of fecal transplant failure. Eur J Gastro Hep 2016;28:826-30.

75 Damman CJ, Brittnacher MJ, Westerhoff $\mathrm{M}$, et al. Low level engraftment and improvement following a single colonoscopic administration of fecal microbiota to patients with ulcerative colitis. PLOS ONE 2015;10:e0133925.

76 Paramsothy S, Kamm M, Walsh A, et al. Multi-donor intense faecal microbiota transplantation is an effective treatment for resistant ulcerative colitis: a randomised placebo-controlled trial [abstract]. J Crohns Colitis 2016;10:S14.

77 Cui $B$, Feng $Q$, Wang $H$, et al. Fecal microbiota transplantation through mid-gut for refractory Crohn's disease: safety, feasibility, and efficacy trial results. J Gastroenterol Hepatol 2015;30:51-8.

78 Khoruts A, Rank KM, Newman KM, et al. Inflammatory bowel disease affects the outcome of fecal microbiota transplantation for recurrent clostridium difficile infection. Clin Gastroenterol Hepatol 2016:14:1433-8.

79 Kelly CR, Ihunnah C, Fischer M, et al. Fecal microbiota transplant for treatment of Clostridium difficile infection in immunocompromised patients. Am J Gastroenterol 2014;109:1065-71.
80 Solari PR, Fairchild PG, Noa LJ, et al. Tempered enthusiasm for fecal transplant. Clin Infect Dis 2014:59:319.

81 Alang N, Kelly CR. Weight gain after fecal microbiota transplantation. Open Forum Infect Dis 2015:2:ofv004.

82 Baxter M, Colville A. Adverse events in faecal microbiota transplant: a review of the literature. J Hosp Infect 2016:92:117-27.

83 Satokari R, Fuentes S, Mattila E, et al. Fecal transplantation treatment of antibiotic-induced, noninfectious colitis and long-term microbiota follow-up. Case Rep Med 2014;2014:913867

84 Moschen AR, Gerner RR, Wang J, et al. Lipocalin 2 protects from inflammation and tumorigenesis associated with gut microbiota alterations. Cell Host Microbe 2016;19:455-69.

85 Wadhwa A, Al Nahhas MF, Dierkhising RA, et al. High risk of post-infectious irritable bowel syndrome in patients with Clostridium difficile infection. Aliment Pharmacol Ther 2016:44:576-82.

86 Sofi AA, Georgescu C, Sodeman T, et al. Physician outlook toward fecal microbiota transplantation in the treatment of Clostridium difficile infection. Am J Gastroenterol 2013;108:1661-2.

87 Bakken JS, Polgreen PM, Beekmann SE, et al. Treatment approaches including fecal microbiota transplantation for recurrent Clostridium difficile infection (RCDI) among infectious disease physicians. Anaerobe 2013;24:20-4.

88 Dennis M, Salpeter MJ, Hota S. Low awareness but positive attitudes toward fecal transplantation in Ontario physicians. Can J Infect Dis Med Microbiol 2015;26:30-2.

89 Ren RR, Sun G, Yang YS, et al. Chinese physicians' perceptions of fecal microbiota transplantation. World J Gastroenterol 2016:22:4757-65.

90 Miller JM, Astles R, Baszler T, et al., Biosafety Blue Ribbon Panel; Centers for Disease Control and Prevention (CDC). Guidelines for safe work practices in human and animal medical diagnostic laboratories. Recommendations of a CDC-convened, Biosafety Blue Ribbon panel. MMWR Supp/ 2012;61:1-102.

91 Good clinical laboratory practice (GCLP). World Health Organization on behalf of the Special Programme for Research and Training in Tropical Diseases, 2009:1-28. http:/ www.who.int/tdr/publications/documents/gclp-web.pdf (accessed 31 Aug 2016).

92 DAIDS Guidelines for Good Clinical Laboratory Practice Standards. 09 July 2013. 1-105. http://www.niaid.nih.gov/LabsAndResources/resources/DAIDSClinRsrch/ Documents/gclp.pdf (accessed 31 Aug 2016).

93 American Gastroenterological Association Center for Gut Microbiome Research \& Education. Center establishes NIH-funded registry to track FMT. http://www.gastro. org/about/initiatives/aga-center-for-gut-microbiome-research-education (accessed 5 Oct 2016).

94 Directive 2004/23/EC of the European Parliament and of the Council of 31 March 2004 on setting standards of quality and safety for the donation, procurement, testing, processing, preservation, storage and distribution of human tissues and cells.

95 Holvoet $\mathrm{T}$, Joossens $\mathrm{M}$, Wang J, et al. Assessment of faecal microbial transfer in irritable bowel syndrome with severe bloating. Gut 2016; doi:10.1136/gutjnl-2016312513 [Epub ahead of print: 10 Aug 2016].

96 Kakihana K, Fujioka Y, Suda W, et al. Fecal microbiota transplantation for patients with steroid-resistant/dependent acute graft-versus-host disease of the gut. Blood 2016;128:2083-8. 